\title{
Analytical results for the Coqblin-Schrieffer model with generalized magnetic fields
}

\author{
V.V. Bazhanov ${ }^{1,2}$, S.L. Lukyanov ${ }^{3,4}$ and A.M. Tsvelik ${ }^{5}$ \\ ${ }^{1}$ Department of Theoretical Physics, Research School of Physical Sciences and Engineering, \\ Australian National University, Canberra, ACT 0200, Australia \\ ${ }^{2}$ Research Institute for Mathematical Sciences, Kyoto University, Kyoto 606-8502, Japan. \\ ${ }^{3}$ Department of Physics and Astronomy, Rutgers University, Piscataway, NJ 08855-0849, USA, \\ ${ }^{4}$ L.D. Landau Institute for Theoretical Physics, Chernogolovka, 142432, Russia, \\ ${ }^{5}$ Department of Physics, Brookhaven National Laboratory, Upton, NY 11973-5000, USA
}

(Dated: May 8, 2003)

\begin{abstract}
Using the approach alternative to the traditional Thermodynamic Bethe Ansatz, we derive analytical expressions for the free energy of Coqblin-Schrieffer model with arbitrary magnetic and crystal fields. In Appendix we discuss two concrete examples including the field generated crossover from the $S U(4)$ to the $S U(2)$ symmetry in the $S U(4)$-symmetric model.
\end{abstract}

The advances of nanotechnology have given an additional weight to the old problem of Kondo effect. Artificially manufactured structures such as quantum dots emulate the behavior of "natural" magnetic impurities though on different energy scales. Using technological means one can widely vary parameters of the dots thus getting an access to previously experimentally unexplored regions of the phase diagram.

The most physically transparent situation corresponds to the case when magnetic impurity (or quantum dot) has a perfect symmetry. This, however, is rarely achieved in real systems due to a presence of the crystalline lattice. Let us consider, for instance, magnetic impurities made of rare-earth magnetic ions of $\mathrm{Ce}$ and $\mathrm{Yb}$. In the presence of a strong spin-orbital coupling an $f^{1}(\mathrm{Ce})$ or $f^{13}(\mathrm{Yb})$ orbital is characterized by the total angular momentum $j(j=5 / 2$ for $\mathrm{Ce}$ and $7 / 2$ for $\mathrm{Yb})$ such that an isolated ion has the $S U(n)$ symmetry with $n=2 j+1$. In the crystalline environment this symmetry is broken. The interplay of these effects with the Kondo screening can be studied using the Coqblin-Schrieffer model [1]:

$$
\mathbf{H}=\sum_{k, a} k \mathbf{c}_{k, a}^{\dagger} \mathbf{c}_{k, a}+\frac{J}{V} \sum_{\substack{k, k^{\prime} \\ a, b}} \mathbf{c}_{k, a}^{\dagger} \mathbf{c}_{k^{\prime}, b} \mathbf{f}_{b}^{\dagger} \mathbf{f}_{a}+\sum_{a} H_{a} \mathbf{f}_{a}^{\dagger} \mathbf{f}_{a}(1)
$$

where $\mathbf{c}_{k, a}^{\dagger}, \mathbf{c}_{k, a}$ are creation (annihilation) operators of the conduction electrons partial harmonics with the angular momentum projection $m=j+1-a(a=1, \ldots n)$; $\mathbf{f}_{a}^{\dagger}$ and $\mathbf{f}_{a}$-operators describe the impurity spin and $V$ is the volume of the system. The generalized magnetic field $H_{a}$ originates from crystal fields inherent to the material and the external magnetic field. Since Hamiltonian (1) commutes with the operator

$$
\mathbf{q}=\sum_{a=1}^{n} \mathbf{f}_{a}^{\dagger} \mathbf{f}_{a}
$$

one can assume without loss of generality that

$$
\sum_{a=1}^{n} H_{a}=0
$$

Notice that the cases described above correspond to the sector of the Coqblin-Schrieffer model with the occupation number $q=1$.

Since model (11) has a linear spectrum, it has to be equipped with the ultraviolet (UV) cutoff $\Lambda$ and a consistent removal of the UV divergences requires that the "bare" coupling $g_{0}=n J \rho(0)$ (here $\rho(0)$ is the conduction electron density of states at the chemical potential) be given certain dependence of the cutoff momenta (see, e.g. [2]):

$$
\Lambda \frac{\mathrm{d} g_{0}}{\mathrm{~d} \Lambda}=-g_{0}^{2}+\frac{g_{0}^{3}}{n}+\ldots .
$$

Eq.(3) shows that for positive $g_{0}$ the Coqblin-Schrieffer model acquires a physical energy scale, the Kondo temperature

$$
T_{K} \sim \Lambda g_{0}^{\frac{1}{n}} \mathrm{e}^{-\frac{1}{g_{0}}}
$$

and renormalization trades the bare coupling constant $g_{0}$ for the renormalization group invariant scale $T_{K}$. Therefore the partition functions of the model in the sector with a given occupation number $q, Z_{q}$, actually depends on the dimensionless combinations $T / T_{K}$ and $H_{a} / T_{K}$. Of course, formula (4) does not specify the physical energy scale uniquely and in order to define $T_{K}$ unambiguously we shall impose the conventional normalization condition [3],

$$
\lim _{T \rightarrow 0} C_{1}(T) /\left.T\right|_{H_{a}=0}=\frac{\pi}{3} \frac{n-1}{T_{K}},
$$

where $C_{1}(T)=T \frac{\partial^{2}}{\partial T^{2}}\left(T \log Z_{q=1}\right)$ is the heat capacity of the impurity in the sector with the occupation number $q=1$.

If the fields are weak in comparison with the Kondo temperature (5), the behavior of the system is governed by $T_{K}^{(n)}$ (the Kondo temperature of the fully $S U(n)$ symmetric model) which for rare earth impurities may be as large as several hundred degrees. On the other 
extreme, if the generalized magnetic fields exceed $T_{K}^{(n)}$ and break down the degeneracy to one Kramers doublet, one gets the Kondo temperature of the order of several degrees. As an illustration let us estimate the new Kondo temperature for the case when the original $S U(n)$ symmetry is broken down to $S U(m)$ by the fields $H_{1}, \ldots H_{n-m} \gg T_{K}^{(n)}$. The estimate is easy if all fields $H_{1}, \ldots H_{n-m}$ are of the same order $\bar{H}$. Since $\ln T_{K}^{(n)} \sim$ $-1 / n J \rho(0), \quad \ln T_{K}^{(m)} \sim-1 / m J \rho(0)$ and the dimensionless ground state energy depends only on $H_{a} / T_{K}^{(n)}$, we conclude that the resulting Kondo temperature is

$$
T_{K}^{(m)} \sim \bar{H}\left(T_{K}^{(n)} / \bar{H}\right)^{\frac{n}{m}},
$$

which may easily constitute a scale vastly different from the Kondo temperature of the unperturbed model. It is clear that the detailed behavior in this crossover interval depends on the field ratios $H_{a} / H_{b}$ and it would be highly desirable to have analytic tools to handle a situation with an arbitrary pattern of fields.

Historically the thermodynamics of the CoqblinSchrieffer model has been examined by the method of Thermodynamic Bethe Ansatz (TBA) [3, 4, 5, 6, 7]. Unfortunately the TBA equations corresponding to Eq.(1) are rather complicated to be studied analytically, and until now the majority of results have been obtained by means of their numerical integration (see [8] and references therein).

This work is based on the approach alternative to the TBA. Here we just outline major steps of our analysis and refer the reader to the papers [9, 10, 11, 12] where the method itself was developed. Let us make some remarks on the general spirit of the approach. It uses the fact that integrable impurity models in general and the Coqblin-Schrieffer model in particular can be mapped to the (1+1)-dimensional bulk conformal field theory (CFT) with a non-conformal boundary interaction (see e.g. [13]). From this point of view, Eq. (11) belongs to the class of exactly solvable boundary theories such that the associated boundary state commutes with the infinite set of mutually commutative local integrals of motion of the bulk system. As a matter of fact, one may say that the boundary state "generates" this set in the sense that it admits the asymptotic large-distance expansion in terms of these local integrals. More precisely, the Hilbert space of the bulk CFT associated with Eq.(1) can be classified in accordance with the $W A_{n-1}$-algebra [14] with the central charge $c=n-1$ and the corresponding boundary state commutes with the set of local integrals of motion introduced in [14].

In the approach adopted in this paper the amplitudes of the boundary state are related to monodromy characteristics of certain ordinary linear differential equations. The key ingredient is the equation of the form,

$$
\left\{\left(-\mathrm{i} \partial_{v}+h_{1}\right) \ldots\left(-\mathrm{i} \partial_{v}+h_{n}\right)-\mathrm{e}^{n \theta} \mathrm{e}^{v} v\right\} \Psi=0,
$$

where $\theta$ and $h_{a}$ are some (complex) parameters. For $\Im m(\theta)=\pi / 2$ the equation admits a solution which specified unambiguously by the following asymptotic as $v \rightarrow+\infty$ :

$$
\begin{aligned}
\Psi_{0}(v, \theta) \rightarrow \quad & \left(-\mathrm{ie}^{\theta} v^{\frac{1}{n}} \mathrm{e}^{\frac{v}{n}}\right)^{-\frac{n-1}{2}} \\
& \times \exp \left\{\mathrm{ie}^{\theta}\left(\mathcal{C}+\int_{0}^{v} d u u^{\frac{1}{n}} \mathrm{e}^{\frac{u}{n}}\right)\right\} .
\end{aligned}
$$

Here $\mathcal{C}$ is an arbitrary constant, whose explicit value is not essential for our purposes. Notice that Eq.(7) is invariant under the transformation, $\theta \rightarrow \theta+2 \pi \mathrm{i} / n$, whereas the asymptotic $(8)$ is not. Hence the analytic continuations of $\Psi_{0}$,

$$
\Psi_{q}(v, \theta)=\Psi_{0}(v, \theta+2 \pi \mathrm{i} q / n),
$$

with integers $q$, generate new solutions of Eq.(7). It is possible to show that the Wronskian $W\left[\Psi_{0}, \Psi_{1}, \ldots \Psi_{n-1}\right]$ does not vanish, so that the set $\left\{\Psi_{q}\right\}_{q=0}^{n-1}$ is a fundamental system of solutions of Eq.(17). By virtue of this fact, solution (9) with $q=n$ can be decomposed as

$$
\Psi_{n}(v, \theta)=\sum_{q=0}^{n-1}(-1)^{n-q-1} Z_{q}(\theta+\mathrm{i} \pi q / n) \Psi_{q}(v, \theta) .
$$

Following the line of arguments similar to that of $[9,10]$, it is possible to show that if the parameters $\theta$ and $h_{a}$ are identified with the dimensionless parameters of the Coqblin-Schrieffer model,

$$
\mathrm{e}^{\theta}=\frac{1}{2 n^{\frac{1}{n}} \Gamma(1 / n)} \frac{T_{K}}{T}, \quad h_{a}=\frac{H_{a}}{2 \pi T},
$$

then the function $Z_{q}(\theta)$ appearing in (10) coincides with the analytic continuation of the partition function of this model, $Z_{q}$, for the sector with the occupation number $q$.

The subject of our current interest is the free energies

$$
\mathcal{F}_{q}=-T \log \left(Z_{q}\right)
$$

at the low temperature limit. In particular we study vacuum energies $\mathcal{E}_{q}=\left.\mathcal{F}_{q}\right|_{T=0}$. They can be obtained from the semi-classical (WKB) approximation for Eq. (17). The leading terms in the WKB expansion of the solution $\Psi_{0}$ (8) read,

$$
\begin{aligned}
& \Psi_{0}(v, \theta) \simeq\left(-\mathrm{i} X\left(\mathrm{e}^{\theta} v^{\frac{1}{n}} \mathrm{e}^{\frac{v}{n}}\right)\right)^{-\frac{n-1}{2}} \times \\
& \exp \left\{\mathrm{ie}^{\theta}\left(\mathcal{C}+\int_{0}^{v} \mathrm{~d} u u^{\frac{1}{n}} \mathrm{e}^{\frac{u}{n}}\right)-\right. \\
& \left.\mathrm{i} \int_{v}^{+\infty} \mathrm{d} u(X(Y)-Y)\right|_{\left.Y=\mathrm{e}^{\theta} u^{\frac{1}{n}} \mathrm{e}^{\frac{u}{n}}\right\} .}
\end{aligned}
$$

Here $X=X(Y)$ is the solution of the algebraic equation

$$
Y^{n}=\left(X+h_{1}\right) \ldots\left(X+h_{n}\right),
$$


such that

$$
X(Y) \rightarrow Y \quad \text { as } \quad Y \rightarrow \infty .
$$

In fact, $X=X(Y)$ is a multi-valued function of the argument $Y$ and Eq. (15) uniquely specifies its branch for a sufficiently large $Y$. The latter solution admits a convergent $1 / Y$-power series expansion found by Lagrange 15]:

$$
X(Y)=Y+\frac{1}{n} \sum_{\substack{k=1 \\ k \neq 0(\bmod n)}}^{\infty} I_{k}\left(h_{1}, \ldots h_{n}\right) Y^{-k} .
$$

Here $I_{k}$ are symmetric polynomials given by

$$
\begin{aligned}
& I_{k}\left(h_{1}, \ldots h_{n}\right)=\sum_{\substack{\alpha_{1}, \ldots \alpha_{n-1} \geq 0 \\
2 \alpha_{1}+3 \alpha_{2}+\ldots n \alpha_{n-1}=k+1}} \frac{(-1)^{\alpha_{1}+\ldots+\alpha_{n-1}}}{\alpha_{1} ! \alpha_{2} ! \ldots \alpha_{n-1} !} \\
& \times \frac{\Gamma\left(\alpha_{1}+\ldots+\alpha_{n-1}-\frac{k}{n}\right)}{\Gamma\left(1-\frac{k}{n}\right)} G_{2}^{\alpha_{1}} G_{3}^{\alpha_{2}} \ldots G_{n}^{\alpha_{n-1}}, \quad(17)
\end{aligned}
$$

where we use the following notation for the elementary symmetric polynomials $G_{k}$ :

$$
G_{k}=\sum_{1 \leq a_{1}<a_{2} \ldots<a_{k} \leq n} h_{a_{1}} \ldots h_{a_{k}} .
$$

Note that according to the constraint (2), we have set $G_{1}=0$, and also $G_{2}=-\frac{1}{2} \sum_{a=1}^{n} h_{a}^{2}$.

Using the Lagrange formula (16) and (13) it is possible to show that the vacuum energies admit the following weak-field expansion [16]

$$
\begin{aligned}
\mathcal{E}_{q} & =E_{0} \sin \left(\frac{\pi q}{n}\right)+\frac{T_{K}}{n} \times \\
& \sum_{\substack{k=1 \\
k \neq 0(\text { mod } n)}}^{\infty} C_{k} \sin \left(\frac{\pi k q}{n}\right) I_{k}\left(\frac{H_{1}}{2 \pi T_{K}}, \ldots \frac{H_{n}}{2 \pi T_{K}}\right),
\end{aligned}
$$

where

$$
C_{k}=2^{k+1} k^{k / n} \Gamma^{k}(1 / n) \Gamma(-k / n) .
$$

The first term in (19) is the vacuum energy for zero fields and the value of $E_{0} \sim T_{K}$ is related to the choice of the constant $\mathcal{C}$ in (8) 17]. Notice that $I_{k}$ (17) are homogeneous symmetric polynomials of the variables $h_{a}$ of the degree $k+1$, i.e.

$$
I_{k}\left(\lambda h_{1}, \ldots \lambda h_{n}\right)=\lambda^{k+1} I_{k}\left(h_{1}, \ldots h_{n}\right),
$$

so Eq.(19) can be considered as a power series expansion in $\bar{H} /\left(2 \pi T_{K}\right)$, where $\bar{H}=\sqrt{\frac{1}{n} \sum_{a=1}^{n} H_{a}^{2}}$. Thus if the fields are weak in comparison with the Kondo temperature the behavior of the system is governed by the convergent series (19). At the same time this series has a finite convergence radius and defines the multi-valued function of complex variable $\bar{H}$ (see Appendix for examples).

We have also examined the structure of the low temperature expansion of the free energy (12) and found the following asymptotic series:

$$
\begin{gathered}
\mathcal{F}_{q}=E_{0} \sin \left(\frac{\pi q}{n}\right)+\frac{T_{K}}{n} \sum_{\substack{k=1 \\
k \neq 0(\text { mod } n)}}^{\infty} C_{k} \sin \left(\frac{\pi k q}{n}\right) \\
\times \mathbb{I}_{k}\left(\frac{H_{1}}{2 \pi T}, \ldots \frac{H_{n}}{2 \pi T}\right)\left(\frac{T}{T_{K}}\right)^{k+1}+O\left(T^{\infty}\right) .
\end{gathered}
$$

Here the numerical coefficients $C_{k}$ are the same as in (19) and $\mathbb{I}_{k}\left(h_{1}, \ldots, h_{n}\right)$ is a symmetric polynomial of the degree $k+1$ of the form,

$$
\mathbb{I}_{k}=I_{k}+J_{k},
$$

where $I_{k}$ is given by Eq. (17) and $J_{k}=J_{k}\left(h_{1}, \ldots, h_{n}\right)$ are some symmetric polynomials of the degree $k$. Currently the polynomial $\mathbb{I}_{k}$ are not known in a closed form for arbitrary $k$. Nevertheless they admit a simple algebraic description. As has been mentioned before, the boundary state associated with the Coqblin-Schrieffer model commutes with the infinite set of mutually commutative integrals of motion for the $W A_{n-1}$-algebra with the central charge $c=n-1$. It turns out that the polynomials $\mathbb{I}_{k}$ appearing in Eq.(21) coincide with vacuum eigenvalues of these conserved charges. Therefore they can be calculated purely algebraically for any finite $k$. In particular we found,

$$
\begin{aligned}
& \mathbb{I}_{1}=-G_{2}+\frac{n-1}{24} \\
& \mathbb{I}_{2}=-G_{3} \\
& \mathbb{I}_{3}=-G_{4}+\frac{n-3}{2 n} G_{2}^{2}-\frac{n-3}{8 n} G_{2}+\frac{3(n-1)(n-3)}{640 n} \\
& \mathbb{I}_{4}=-G_{5}+\frac{n-4}{n} G_{3} G_{2}-\frac{n-4}{3 n} G_{3} .
\end{aligned}
$$

Here $G_{k}$ are the elementary symmetric polynomials (18). Notice that for the physically interesting case of the magnetic field configuration

$$
h_{a}=h(j+1-a), \quad j=\frac{n-1}{2},
$$

all polynomials $\mathbb{I}_{k}$ with even $k$ vanish, whereas using Eq. (23) one has:

$$
\begin{aligned}
& \mathbb{I}_{1}=\frac{n-1}{24}\left(n(n+1) h^{2}+1\right), \\
& \mathbb{I}_{3}=\frac{(n-1)(n-3)}{1920 n} \times \\
& \left(n^{2}(n+1)(n+3) h^{4}+10 n(n+1) h^{2}+9\right) .
\end{aligned}
$$

In conclusion let us just repeat the basic results of the paper. We have outlined a method which allows 
to extract analytical results for the thermodynamics of Coqblin-Schrieffer model in the presence of magnetic and crystal fields. The general formulae drastically simplify for the $S U(4)$ model (see Appendix).

Acknowledgments. S.L.L. and A.M.T. are grateful to N. Andrei, F.H.L. Essler and A.B. Zamolodchikov for interesting discussions and constructive criticism. A.M.T. acknowledges the support from US DOE under contract number DE-AC02-98 CH 10886. Research of S.L.L. is supported in part by DOE grant \#DE-FG02-96 ER 40959; he also acknowledges a support from Institute for Strongly Correlated and Complex Systems at BNL.

\section{Appendix}

In this appendix we illustrate the expansion (19) using two examples.

Our first example is related to the case $n=4$. Let us consider the following configuration of the generalized magnetic fields:

$$
H_{1}=-H_{4}, \quad H_{2}=-H_{3},
$$

with $H_{1} \geq H_{2} \geq 0$. In this case Eq. (14) is especially simple and can be written in the form

$$
Y^{4}=\left(X^{2}-h_{1}^{2}\right)\left(X^{2}-h_{2}^{2}\right)
$$

Its solution $X=X(Y)$ satisfying (15) reads explicitly:

$$
x=y \sqrt{\sqrt{1+\epsilon / y^{4}}+1 / y^{2}},
$$

where $y=Y / \bar{h}, x=X / \bar{h}$, and

$$
\bar{h}=\sqrt{\frac{h_{1}^{2}+h_{2}^{2}}{2}}, \quad \epsilon=\left(\frac{h_{1}^{2}-h_{2}^{2}}{h_{1}^{2}+h_{2}^{2}}\right)^{2} .
$$

All even coefficients $I_{2 l}$ in series (16) vanish now, whereas $I_{2 l-1}$ can be expressed in terms of the hypergeometric function:

$$
I_{2 l-1}=2(-1)^{l-1} \frac{\Gamma\left(l-\frac{1}{2}\right)}{\sqrt{\pi} l !} \bar{h}^{2 l}{ }_{2} F_{1}\left(-\frac{l}{2}, \frac{1}{2}-\frac{l}{2}, \frac{3}{4}-\frac{l}{2} ; \epsilon\right) .
$$

Combining this equation with (19) one obtains,

$$
\begin{aligned}
& \mathcal{E}_{q}=E_{0} \sin \left(\frac{\pi q}{4}\right)+\frac{T_{K}}{\sqrt{2} \Gamma(1 / 4)} \times \\
& \sum_{l=1}^{\infty} \frac{(-1)^{l}}{l !}(2 l-1)^{\frac{l}{2}-\frac{5}{4}} \Gamma\left(\frac{l}{2}+\frac{1}{4}\right) \frac{\sin \left(\frac{\pi(2 l-1) q}{4}\right)}{\sin \left(\frac{\pi(2 l-1)}{4}\right)} \times \\
& { }_{2} F_{1}\left(-\frac{l}{2}, \frac{1}{2}-\frac{l}{2}, \frac{3}{4}-\frac{l}{2} ; \epsilon\right)\left(\frac{\Gamma(1 / 4) \sqrt{2} \bar{H}}{\pi T_{K}}\right)^{2 l},
\end{aligned}
$$

with

$$
\bar{H}=\sqrt{\frac{H_{1}^{2}+H_{2}^{2}}{2}}, \quad \epsilon=\left(\frac{H_{1}^{2}-H_{2}^{2}}{H_{1}^{2}+H_{2}^{2}}\right)^{2} \leq 1 .
$$

Eq.(30) can be rewritten in the form of a convergent integral which is useful for an analytical continuation of the power series expansion outside its convergence disk,

$$
\left|\bar{H} / T_{K}\right|<\frac{\pi \mathrm{e}^{-\frac{1}{4}}}{\sqrt{2} \Gamma(1 / 4)} \min \left[\epsilon^{-\frac{1}{4}},(1-\epsilon)^{-\frac{1}{4}}\right] .
$$

Explicitly one has $(q=1,2,3)$ :

$$
\begin{aligned}
& \mathcal{E}_{q}=E_{0} \sin \left(\frac{\pi q}{4}\right)+\bar{H} \sin \left(\frac{\pi q}{4}\right) \times \\
& \int_{-\infty}^{\infty} \frac{\mathrm{d} \omega}{2 \pi}(\mathrm{i} \omega+0)^{\mathrm{i} \omega-1} \frac{\Gamma\left(-\frac{1}{2}-2 \mathrm{i} \omega\right)}{\Gamma\left(\frac{1}{2}-\mathrm{i} \omega\right)}\left(\frac{2 \Gamma(1 / 4) \bar{H}}{\pi T_{K}}\right)^{4 \mathrm{i} \omega} \times \\
& { }_{2} F_{1}\left(\frac{1}{4}-\mathrm{i} \omega,-\frac{1}{4}-\mathrm{i} \omega, \frac{1}{2}-\mathrm{i} \omega ; \frac{1}{2}-(-1)^{q} \frac{1-2 \epsilon}{2}\right) .
\end{aligned}
$$

It is particularly illuminating to extract from this expression the Kondo temperature for the $S U(2)$ CoqblinSchrieffer model to compare it with Eq.(6). The Kondo temperature $T_{K}^{(2)}$ can be extracted from the magnetic susceptibility in the limit when one of the fields is very large. Since the energy depends on two fields $H_{1}, H_{2}$, one has to be careful in choosing the right direction of differentiation. The right choice of variables is $H_{ \pm}=$ $H_{1} \pm H_{2}$ such that $\partial \mathcal{E}_{1} / \partial H_{-}=0$ at $H_{-}=0$. Then at $H_{+} \rightarrow 2 \bar{H} \gg T_{K}, H_{-} \rightarrow 0$ we obtain for $q=1$ the following expression for the magnetic susceptibility

$$
\begin{aligned}
& \chi=-\left.\frac{\partial^{2} \mathcal{E}_{1}}{\partial H_{-}^{2}}\right|_{\substack{H_{-}=0 \\
H_{+}=2 \bar{H}}} \rightarrow \frac{1}{2 \pi T_{K}^{(2)}}, \\
& T_{K}^{(2)}=\frac{\pi^{3 / 2}}{\sqrt{8} \Gamma^{2}(1 / 4)} \frac{T_{K}^{2}}{\bar{H}} .
\end{aligned}
$$

The above expression for the effective Kondo temperature is a particular case of Eq.(6) for $n=4, m=2$.

Our second example is related to the case of an arbitrary integer $n$. In [3], the vacuum energy $\mathcal{E}_{1}$ was found for the following field configuration,

$$
H_{a}=\sqrt{2} \bar{H} \cos \left(\frac{\pi(2 a-1)}{2 n}\right) .
$$

For this pattern the equation (14) is expressed in terms of the Chebyshev polynomials $T_{n}(x)=\cos (n \arccos (x))$ :

$$
y^{n}=2^{1-\frac{n}{2}} T_{n}(x / \sqrt{2}),
$$

where $y=Y / \bar{h}, x=X / \bar{h}$. Therefore

$$
\begin{aligned}
x=\quad & y_{2} F_{1}\left(-\frac{1}{2 n}, \frac{n-1}{2 n}, \frac{n-1}{n} ; \frac{2^{2-n}}{y^{2 n}}\right)+ \\
& \frac{1}{2 y}{ }_{2} F_{1}\left(\frac{1}{2 n}, \frac{n+1}{2 n}, \frac{n+1}{n} ; \frac{2^{2-n}}{y^{2 n}}\right),
\end{aligned}
$$

and non-vanishing coefficients in (16) and (19) are $I_{2 n l-1}(l=1,2 \ldots)$ and $I_{2 n l+1}(l=0,1,2, \ldots)$ :

$$
I_{2 n l+\sigma}=\sigma \frac{\Gamma\left(\frac{\sigma}{n}+2 l\right)}{l ! \Gamma\left(1+\frac{\sigma}{n}+l\right)}\left(\frac{\bar{h}}{\sqrt{2}}\right)^{2 l n+1+\sigma}, \sigma= \pm 1 .
$$


As in the first example, the vacuum energies (19) can be written in the form of convergent integral [3]:

$$
\begin{aligned}
& \mathcal{E}_{q}=E_{0} \sin \left(\frac{\pi q}{n}\right)+ \\
& \frac{\sqrt{2} \bar{H} \sin (\pi q / n)}{4 \pi n} \int_{-\infty}^{\infty} \frac{\mathrm{d} \omega}{2 \pi}(\mathrm{i} \omega+0)^{\mathrm{i} \omega-1} \times \\
& \Gamma\left(-\frac{1}{2 n}-\frac{\mathrm{i} \omega}{2}\right) \Gamma\left(\frac{1}{2 n}-\frac{\mathrm{i} \omega}{2}\right)\left(\frac{n^{\frac{1}{n}} \Gamma(1 / n) \bar{H}}{\pi \sqrt{2} T_{K}}\right)^{\mathrm{i} n \omega} .
\end{aligned}
$$

It is easy to check that this equation for $n=4$ is in agreement with (31) provided $\epsilon=1 / 2$.

[1] B. Coqblin and J.R. Schrieffer, Phys. Rev. 185, 847 (1969).

[2] N. Read and D.N. Newns, J. Phys. C16, 3273 (1983).

[3] A.M. Tsvelik and P.B. Wiegmann, J. Phys. C15, 1707 (1982); Adv. in Phys. 32, 453 (1983).

[4] N. Andrei, K. Furuya and J.H. Lowenstein, Rev. Mod. Phys. 55, 331 (1983).

[5] J.W. Rasul, in Proceedings of the International Conference on Valence Fluctuations, Zurich, Switzerland, eds. P. Wachter and H. Boppart, North-Holland, Amsterdam (1982).

[6] P. Coleman and N. Andrei, J. Phys. C19, 3211 (1986).
[7] P. Schlottmann, Z. Phys. B55, 293 (1984); Phys. Rev. B30, 1454 (1984).

[8] P. Schlottmann, Phys. Rep. 181, 1 (1989).

[9] V.V. Bazhanov, S.L. Lukyanov and A.B. Zamolodchikov, Comm. Math. Phys. 177, 381 (1996); 190, 247 (1997); 200, 297 (1999); Jour. Stat. Phys. 102, 567 (2001).

[10] V.V. Bazhanov, A.N. Hibberd and S.M. Khoroshkin, Nucl.Phys. B622, 475 (2002).

[11] P. Dorey and R. Tateo, J. Phys. A32 L419 (1999); A33 8427 (2000).

[12] S.L. Lukyanov and A.B. Zamolodchikov, Integrable spherical brane model and Coulomb charging at large conduction, to appear.

[13] I. Affleck, Acta Phys. Polon. B26, 1869 (1995).

[14] S. Lukyanov and V. Fateev, Sov. Sci. Rev. A. Phys. 15 pp.1-117 (1990).

[15] J.L. Lagrange, Mémoires de l'Académie de Berlin, (1768). G. Belardinelli, Fonctions hypergéométriques de plusieurs variables et résolution analytique des équations algébriques générales, Ser.: Mémor. Sci. Math., Fasc. 145, Gauthier-Villars, Paris, 1960, p.50.

[16] Using the result of H.J. Mellin, Ann. Ac. Sc. Fenn. 7 no. 8 (1915); C. R. Acad. Sc. 172 (1921) 658, one can rewrite (19) as a multiple Mellin-Barns integral convenient for its analytic continuation in the variables $H_{a}$ beyond the convergence domain of the sum (19).

[17] According to the $1 / n$-expansion of Ref.[2], $E_{0} / T_{K}=1+$ $O\left(n^{-2}\right)$. 